\title{
A Multi Item Integrated Inventory Model with Reparability and Manufacturing of Fresh Products
}

\author{
Pinky Saxena ${ }^{1}$, S. R. Singh ${ }^{2} \&$ Isha Sangal ${ }^{3}$ \\ ${ }^{1}$ Department of Mathematics, Krishna Institute of Engineering and Technology, Ghaziabad, India \\ ${ }^{2}$ Department of Mathematics, C.C.S University, Meerut, India \\ ${ }^{3}$ Center for Mathematical Sciences, Banasthali University, Rajasthan, India \\ Correspondence: S. R Singh, Department of Mathematics, C.C.S University, Meerut, 250001, India. Tel: \\ 98-9768-2738. E-mail: shivrajpundir@gmail.com
}

Received: October 18, 2015

Accepted: March 5, 2016

Online Published: April 28, 2016

doi:10.5539/mas.v10n7p74

URL: http://dx.doi.org/10.5539/mas.v10n7p74

\begin{abstract}
In this paper a multi item integrated inventory model is presented with reparability of returned items. It is assumed here that only a certain ratio of returned items can be repaired and the remaining stock of returned items is salvaged. By using these returned items, the waste can be reduced, which pollute the environment. This is a green supply chain where the demand for the products is selling price dependent and production rate is taken as a function of demand rate. The shortages are allowed here. A numerical example and sensitivity analysis are also presented to illustrate the model.
\end{abstract}

Keywords: integrated inventory, reparability, multi items, deterioration, shortages

\section{Introduction}

In so many industrial systems, there is more than one manufacturing plant and they produce different type of items. Most of the classical inventory system tries to calculate the economic production quantity by which the total cost of the system can be optimized. But these models are developed only for single item. In this model we have presented an integrated inventory model for multi items. Considering multi items in inventory model, (Ben-Daya \& Raouf, 1993) have developed an approach for more realistic and general concept of budgetary and floor constraints, where the demand of items follows a uniform probability distribution function. A multi item inventory model for deteriorating items is developed by (Bhattacharya, 2005). (Roy et al., 1995) developed multi deteriorating items with constraint space and investment and obtained some interesting results.( Balkhi ,2009) has introduced a general model for multi item production inventory system in which the cost parameters are treated as an arbitrary function of time. (Yadav et al., 2011) developed a fuzzy multi item production inventory model with reliability and flexibility under limited storage capacity with deterioration via geometric programming. (Singhal \& Singh, 2013) introduced a volume flexible multi items inventory system with imprecise environment. (Tayal et al., 2014) presented a multi item inventory model for deteriorating items with expiration date and allowable shortages.

However, in most of the classical production inventory models even concerned with multi items the attention is given only for the optimality of separate member of the integrated system. For any successful supply chain coordination between the vendor and the buyer is required. This close relationship between vendor and buyer is a key of success for any business organization. Then, a new approach of integration of all the functions in a supply chain was identified.( Singh \& Diksha ,2009) developed an integrated cooperative inventory model for vendor and buyer under progressive credit period in which demand is assumed to be a multivariate function. (Hadidi et al., 2011) developed an integrated production inventory model for scheduling and perfect maintenance. This work integrates, simultaneously, the decisions of preventive maintenance and job order sequencing for a single machine.( Soni \& Patel,2014) presented an optimal decision policy for integrated vendor-buyer inventory system concerning defective items with variable lead time and service level constraint. (Tayal et al, 2016) introduced an integrated production inventory model for perishable products with trade credit period and investment in preservation technology.

Concerning the deterioration and reparability in an inventory model is more realistic and general concept. (Park, 
1983) studied a production inventory system for a single product with deteriorating raw materials. (Yan \& Cheng, 1998) introduced an optimal production stopping and restarting times for an EOQ model with deteriorating items.

(Maity \& Maiti, 2009) developed optimal inventory policies for deteriorating complementary and substitute items. (Singh et al., 2010) presented an EOQ model with Pareto distribution for deterioration, Trapezoidal type demand and backlogging under trade credit policy. Singh et al. (2013) introduced an EOQ model with volume agility, variable demand rate, Weibull deterioration rate and inflation. (Tayal et al.,2014) developed a two echelon supply chain model for deteriorating items with effective investment in preservation technology after that (Tayal et al. ,2014) studied an inventory model for deteriorating items with seasonal products and an option of an alternative market. (Singh et al., 2015) presented an EPQ inventory model for non-instantaneous deteriorating item with time dependent holding cost and exponential demand rate. (Singh et al., 2016) developed an economic order quantity model for deteriorating products having stock dependent demand with trade credit period and preservation technology. One of the first authors was (Schrady, 1967) who developed a simple heuristic procedure for determining the lot sizes of remanufacturing and manufacturing lots. He proposed a simple EOQ-technique that optimizes the sum of fixed and holding costs per time unit. (Teunter.2001) generalized the results of Schrady in a way that he examined different structures of a remanufacturing cycle. His analysis concludes that it is not efficient if more than one remanufacturing lot and more than one manufacturing lot are established in the same repair cycle. (Saxena et.al. 2013) presented two-warehouse production inventory model with variable demand and permissible delay in payment under inflation. (Saxena et.al.2014) generalized production model under fuzzy environment. They compared both the results obtained considering crisp data as well as fuzzy data. (Singh \& Singh, 2013) presented green supply chain model with product remanufacturing under volume flexible environment. (Singh \& Prasher, 2014) introduced a production inventory model with flexible manufacturing, random machine breakdown and stochastic repair time.

In this paper we have presented an integrated inventory model for multi items in a closed loop supply chain with reparability of returned and collected items. The shortages are allowed for retailer and assumed to be completely lost. The repaired items are assumed to be equivalent to fresh products.

\section{Assumptions and Notations}

\subsection{Assumptions}

1. This is a multi item inventory model, presented for the integrated production of new products and reparability of collected items.

2. The demand for the products is a function of selling price.

3. The products after reparability are assumed to be equivalent to new products.

4. The production rate is assumed to be a function of demand rate.

5. The used items are collected at a rate of $b\left(\alpha_{i}-\beta_{i} s_{i}\right)$.

6. A certain ratio $\gamma_{\mathrm{i}}$ of collected items, whose quality level is acceptable for reparability, is used for production and rest of the material is salvaged.

7. The items are assumed to be deteriorating in nature.

8. The shortages are allowed for retailer and occurring shortages are assumed to be completely lost.

\subsection{Notations}

$$
\begin{array}{ll}
\theta & \text { deterioration rate } \\
\alpha_{\mathrm{i}}, \beta_{\mathrm{i}} & \text { demand parameters for } \mathrm{i}^{\text {th }} \text { item } \\
\mathrm{s}_{\mathrm{i} 1}, \mathrm{~s}_{\mathrm{i} 2} & \text { selling price per unit for the producer and the retailer for } \mathrm{i}^{\text {th }} \text { item } \\
\mathrm{a}_{\mathrm{i}} & \quad \text { production parameter for } \mathrm{i}^{\text {th }} \text { item, } \mathrm{a} \geq 1 \\
\mathrm{~b}_{\mathrm{i}} & \quad \text { collection parameter for } \mathrm{i}^{\text {th }} \text { item, } \mathrm{b}<1 \\
& \mathrm{t}_{1 \mathrm{i}} \quad \text { time for remanufacturing for } \mathrm{i}^{\text {th }} \text { item } \\
\mathrm{t}_{2 \mathrm{i}} & \text { time at which inventory level for remanufactured } \mathrm{i}^{\text {th }} \text { item becomes zero } \\
\mathrm{t}_{3 \mathrm{i}} & \text { time up to which production of fresh } \mathrm{i}^{\text {th }} \text { items occur } \\
\mathrm{T}_{\mathrm{i}} & \text { length of the complete cycle for } \mathrm{i}^{\text {th }} \text { item } \\
\mathrm{I}_{\mathrm{ri}}(\mathrm{t}) \text { inventory level for remanufactured } \mathrm{i}^{\text {th }} \text { item at any time } \mathrm{t}
\end{array}
$$


$I_{m i}(t)$ inventory level for fresh produced $i^{\text {th }}$ item at any time $t$

$I_{R i}(t)$ inventory level of collected $i^{\text {th }}$ item at any time $t$

$I_{\text {si }}(t)$ retailer's inventory level for $i^{\text {th }}$ item at any time $t$

$v_{i}$ the time at which the inventory level of $i^{\text {th }}$ item becomes zero for retailer

$n$ number of replenishment cycles for the retailer

$\mathrm{c}_{\mathrm{mi}}$ procurement cost per unit for $\mathrm{i}^{\text {th }}$ item

$c_{R i}$ acquisition cost per unit for $i^{\text {th }}$ item

$\mathrm{P}_{\mathrm{mi}}$ production cost per unit for ith item

$P_{\text {ri }}$ remanufacturing cost per unit for $i^{\text {th }}$ item

$\mathrm{h}_{\mathrm{ri}}, \mathrm{h}_{\mathrm{mi}}, \mathrm{h}_{\mathrm{Ri}}, \mathrm{h}_{\mathrm{si}}$ holding cost per unit for $\mathrm{i}^{\text {th }}$ item for remanufactured items, produced items, collective items and for the retailer

$\mathrm{O}_{\mathrm{i}}$ ordering cost per order

$c_{1 i} \quad$ purchasing cost per unit for $i^{\text {th }}$ item for the retailer

$c_{2 i} \quad$ lost sale cost cost per unit for $\mathrm{i}^{\text {th }}$ item for the retailer

$\mathrm{K}_{1 \mathrm{i}}, \mathrm{K}_{2 \mathrm{i}}, \mathrm{K}_{3 \mathrm{i}}$ set up cost for remanufacturing, fresh production and for the collection

$\mathrm{I}_{1 \mathrm{i}}(0) \quad$ initial inventory level for the retailer for $\mathrm{i}^{\text {th }}$ item

$\mathrm{Q}_{2 \mathrm{i}} \quad$ shortage amount for $\mathrm{i}^{\text {th }}$ item

\section{Mathematical Model}

In this the used items are collected from the market and a certain ratio of these items is repaired. In the above mentioned figure the remanufacturing graph is shown. During $\left[0, t_{1 i}\right]$ remanufacturing occurs and the inventory level of collected items decreases and becomes zero at time $t_{1 i}$. During $\left[t_{1 i}, t_{2 i}\right]$ the inventory level of repaired items depletes due to combined effect of demand and deterioration. After $t=t_{1 i}$ the inventory level of collected items again piled up. At $t=t_{2 i}$ the manufacturing of fresh products start and occurs up to $t=t_{3 i}$. The below mentioned figure (1) shows the inventory level of this system.

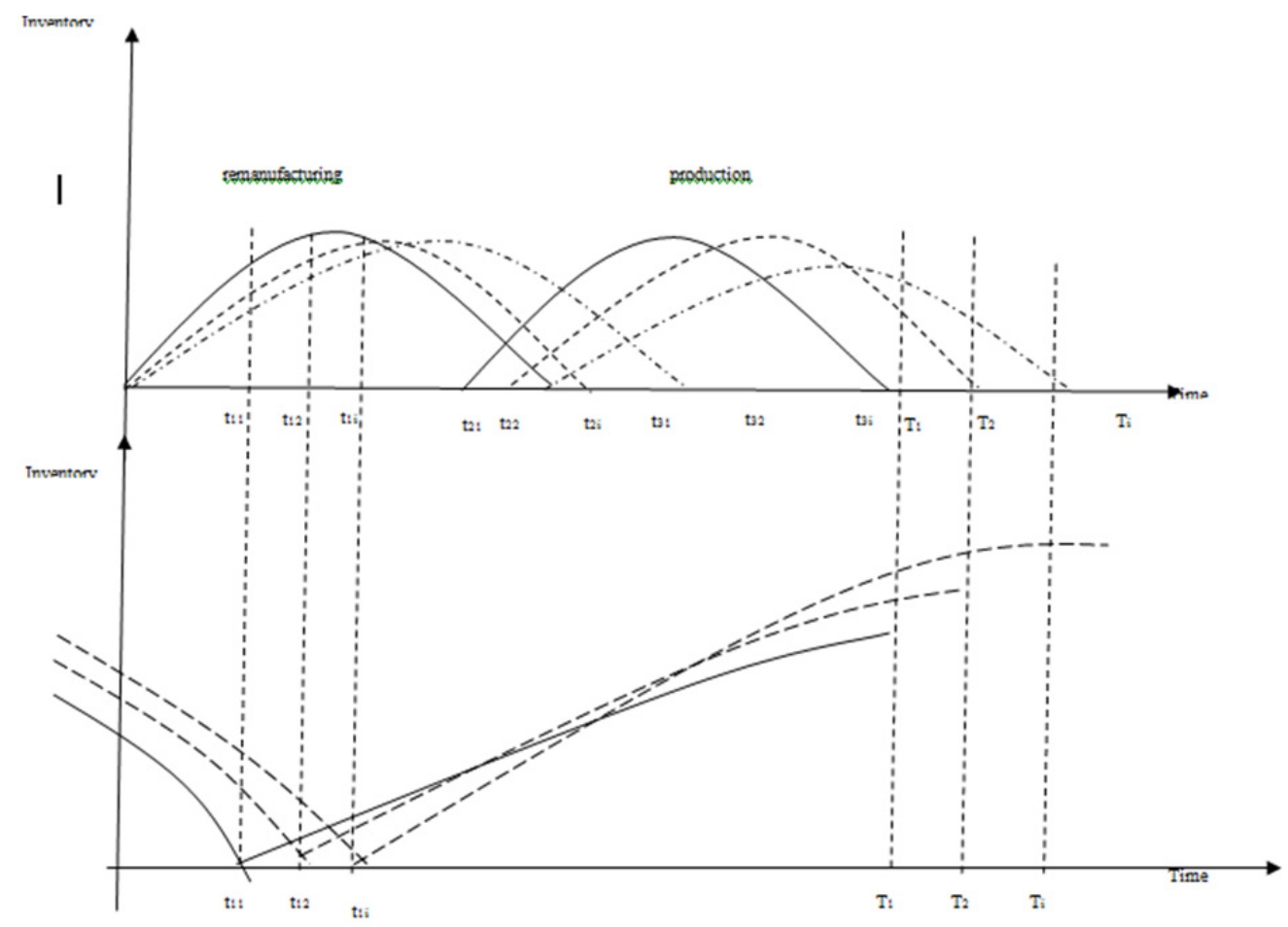

Figure 1. Inventory time behavior for repaired items, fresh produced items and returned items 
These are the differential equations showing the inventory with the variation in time for repaired items, fresh production and collected items.

$$
\begin{array}{ll}
\frac{d I_{r i}(t)}{d t}+\theta I_{r i}(t)=(a-1)\left(\alpha_{i}-\beta_{i} s_{i 1}\right) & 0 \leq t \leq t_{1 i} \\
\frac{d I_{r i}(t)}{d t}+\theta I_{r i}(t)=\left(\alpha_{i}-\beta_{i} s_{i 1}\right) & t_{1 i} \leq t \leq t_{2 i} \\
\frac{d I_{m i}(t)}{d t}+\theta I_{m i}(t)=(a-1)\left(\alpha_{i}-\beta_{i} s_{i 1}\right) & t_{2 i} \leq t \leq t_{3 i} \\
\frac{d I_{m i}(t)}{d t}+\theta I_{m i}(t)=\left(\alpha_{i}-\beta_{i} s_{i 1}\right) & t_{3 i} \leq t \leq T_{i} \\
\frac{d I_{R i}(t)}{d t}+\theta I_{R i}(t)=(b-1)\left(\alpha_{i}-\beta_{i} s_{i 1}\right) & 0 \leq t \leq t_{1 i} \\
\frac{d I_{R i}(t)}{d t}+\theta I_{R i}(t)=b\left(\alpha_{i}-\beta_{i} s_{i 1}\right) & t_{1 i} \leq t \leq T_{i}
\end{array}
$$

with boundary conditions:

$$
I_{r i}(0)=0, I_{r i}\left(t_{2 i}\right)=0, I_{m i}\left(t_{2 i}\right)=0, I_{m i}\left(T_{i}\right)=0, I_{R i}\left(t_{1 i}\right)=0, I_{R i}(0)=I_{R i}\left(T_{i}\right)
$$

The solution of these above mentioned equations are given as follow:

$$
\begin{array}{rlrl}
I_{r i}(t) & =\frac{(a-1)}{\theta}\left(\alpha_{i}-\beta_{i} s_{i 1}\right)\left(1-e^{-\theta t}\right) & 0 \leq t \leq t_{1 i} \\
I_{r i}(t) & =\frac{\left(\alpha_{i}-\beta_{i} s_{i 1}\right)}{\theta}\left(e^{\theta\left(t_{2 i}-t\right)}-1\right) & t_{1 i} \leq t \leq t_{2 i} \\
I_{m i}(t)=\frac{(a-1)}{\theta}\left(\alpha_{i}-\beta_{i} s_{i 1}\right)\left(1-e^{\theta\left(t_{2 i}-t\right)}\right) & t_{2 i} \leq t \leq t_{3 i} \\
I_{m i}(t)=\frac{\left(\alpha_{i}-\beta_{i} s_{i 1}\right)}{\theta}\left(e^{\theta\left(T_{i}-t\right)}-1\right) & t_{3 i} \leq t \leq T_{i} \\
I_{R i}(t)=\frac{\left(\alpha_{i}-\beta_{i} S_{i 1}\right)}{\theta}(b-a)\left(1-e^{\theta\left(t_{1 i}-t\right)}\right) & 0 \leq t \leq t_{1 i} \\
I_{R i}(t)=\frac{b}{\theta}\left(\alpha_{i}-\beta_{i} S_{i 1}\right)\left(1-e^{\theta\left(t_{i i}-t\right)}\right) & t_{1 i} \leq t \leq T_{i}
\end{array}
$$

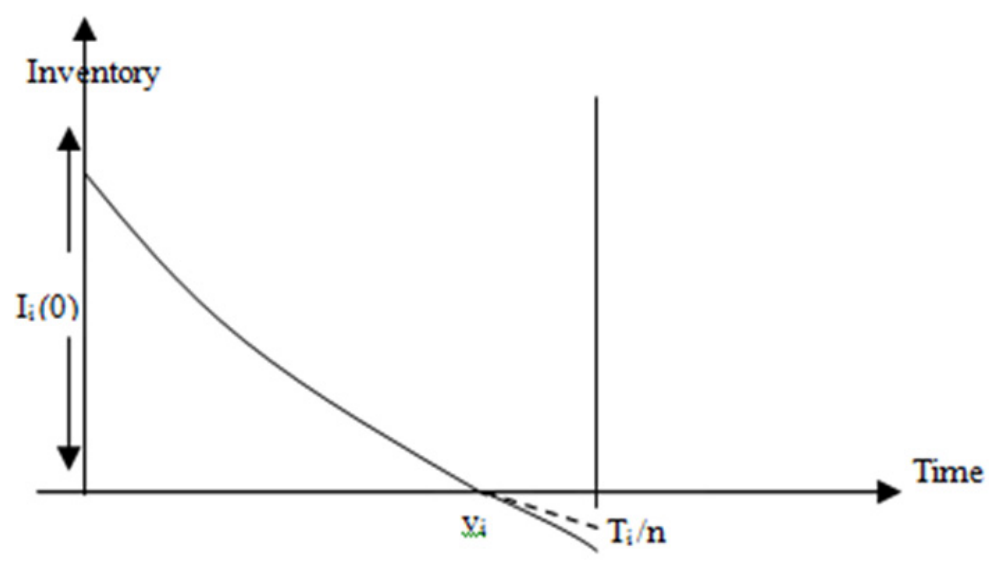

Figure 2. Retailer's inventory 
If $\mathrm{I}_{\mathrm{si}}(\mathrm{t})$ denotes the retailer's inventory level for ith item at any time $t$, then the differential equations showing the inventory level at any time $t$ are given as follow:

$$
\begin{array}{ll}
\frac{d I_{s i}(t)}{d t}+\theta I_{s i}(t)=-\left(\alpha_{i}-\beta_{i} s_{i 2}\right) & 0 \leq t \leq v_{i} \\
\frac{d I_{s i}(t)}{d t}=-\left(\alpha_{i}-\beta_{i} s_{i 2}\right) & v_{i} \leq t \leq \frac{T_{i}}{n}
\end{array}
$$

with boundary condition $I_{s i}\left(v_{i}\right)=0$

The solution of these above mentioned equations are given as follow:

$$
\begin{array}{ll}
I_{s i}(t)=\frac{\left(\alpha_{i}-\beta_{i} s_{i 2}\right)}{\theta}\left(e^{\theta\left(v_{i}-t\right)}-1\right) & 0 \leq t \leq v_{i} \\
I_{s i}(t)=\left(\alpha_{i}-\beta_{i} s_{i 2}\right)\left(v_{i}-t\right) & v_{i} \leq t \leq \frac{T_{i}}{n}
\end{array}
$$

The total average cost for the ith item for the manufacturer is given by:

T.A.C $C_{m i}=\frac{1}{T_{i}}[$ Procurement cost + Acquisition cost + Production cost +

$$
\text { Remanufacturing cost }+ \text { Holding cost }+ \text { Salvage cost }+ \text { Set up cost }]
$$

The total average cost for the ith item for the retailer is given by:

$$
\text { T.A.C } \left.C_{s i}=\frac{n}{T_{i}} \text { [Purchasing cost }+ \text { Holding cost }+ \text { ordering cost }+ \text { lost sale cost }\right]
$$

\section{Different associated costs for manufacturer:}

$$
\begin{gathered}
\text { Procurement cost }=c_{m i} \int_{t_{2 i}}^{t_{3 i}} a\left(\alpha_{i}-\beta_{i} s_{i 1}\right) d t \\
\text { Procurement cost }=c_{m i} a\left(\alpha_{i}-\beta_{i} s_{i 1}\right)\left(t_{3 i}-t_{2 i}\right) \\
\text { Acquisition cost }=c_{R i} \int_{0}^{T_{i}} b\left(\alpha_{i}-\beta_{i} s_{i 1}\right) d t \\
\text { Acquisition cost }=c_{R i} b T_{i}\left(\alpha_{i}-\beta_{i} s_{i 1}\right) \\
\text { Production cost }=P_{m i} a \int_{t_{2 i}}^{t_{3 i}}\left(\alpha_{i}-\beta_{i} s_{i 1}\right) d t \\
\text { Production cost }=P_{m i} a\left(t_{3 i}-t_{2 i}\right)\left(\alpha_{i}-\beta_{i} s_{i 1}\right) \\
\text { Remanufacturing cost }=P_{r i} a \int_{0}^{t_{1 i}}\left(\alpha_{i}-\beta_{i} s_{i 1}\right) d t \\
\text { Remanufacturing cost }=P_{r i} a t_{1 i}\left(\alpha_{i}-\beta_{i} s_{i 1}\right) \\
\text { Set up cost }=K_{1 i}+K_{2 i}+K_{3 i} \\
\text { Salvage cost }=s_{v i}\left\{\left(1-\gamma_{i}\right) b\left(\alpha_{i}-\beta_{i} s_{i 1}\right)\right\} T_{i}
\end{gathered}
$$

Holding cost $=$

$$
h_{r i} \int_{0}^{t_{1 i}} I_{r i}(t) d t+h_{r i} \int_{t_{1 i}}^{t_{2 i}} I_{r i}(t) d t+h_{m i} \int_{t_{2 i}}^{t_{3 i}} I_{m i}(t) d t+h_{m i} \int_{t_{3 i}}^{T_{i}} I_{m i}(t) d t+h_{R i} \int_{0}^{t_{1 i}} I_{R i}(t) d t+h_{R i} \int_{t_{i i}}^{T_{i}} I_{R i}(t) d t
$$


Holding $\cos \mathrm{t}=$

$$
\begin{aligned}
& h_{r i}\left\{\frac{(a-1)}{\theta}\left(\alpha_{i}-\beta_{i} s_{i 1}\right)\left(t_{1 i}+\frac{e^{-\theta t_{1 i}}-1}{\theta}\right)+\frac{\left(\alpha_{i}-\beta_{i} s_{i 1}\right)}{\theta}\left(\frac{e^{\theta\left(t_{2 i}-t_{1 i}\right)}-1}{\theta}\right)+\left(t_{1 i}-t_{2 i}\right)\right\} \\
& +h_{m i}\left\{\frac{(a-1)}{\theta}\left(\alpha_{i}-\beta_{i} s_{i 1}\right)\left\{\left(t_{3 i}-t_{2 i}\right)+\frac{e^{\theta\left(t_{2 i}-t_{3 i}\right)}-1}{\theta}\right\}+\frac{\left(\alpha_{i}-\beta_{i} s_{i 1}\right)}{\theta}\left\{\left(\frac{e^{\theta\left(T_{i}-t_{3 i}\right)}-1}{\theta}\right)-\left(T_{i}-t_{3 i}\right)\right\}\right\} \\
& \quad+h_{R i}\left\{\frac{(b-a)}{\theta}\left(\alpha_{i}-\beta_{i} s_{i 1}\right)\left(t_{1 i}-\frac{1-e^{\theta t_{1 i}}}{\theta}\right)+b \frac{\left(\alpha_{i}-\beta_{i} s_{i 1}\right)}{\theta}\left\{\left(\frac{e^{\theta\left(t_{1 i}-T_{i}\right)}-1}{\theta}\right)+\left(T_{i}-t_{1 i}\right)\right\}\right\}
\end{aligned}
$$

\section{Different associated costs for retailer:}

Purchasing cost $=\left(I_{1 i}(0)+Q_{2 i}\right) c_{1 i}$

where

$$
\begin{aligned}
I_{1 i}(0) & =\frac{\left(\alpha_{i}-\beta_{i} s_{i 2}\right)}{\theta}\left(e^{\theta v_{i}}-1\right) \\
Q_{2} & =\int_{v_{i}}^{\frac{T_{i}}{n}}\left(\alpha_{i}-\beta_{i} s_{i 2}\right) \eta d t \\
\text { P.C. } & =\left\{\frac{\left(\alpha_{i}-\beta_{i} s_{i 2}\right)}{\theta}\left(e^{\theta v_{i}}-1\right)+\left(\alpha_{i}-\beta_{i} s_{i 2}\right) \eta\left(\frac{T_{i}}{n}-v_{i}\right)\right\} c_{1 i}
\end{aligned}
$$

$$
\begin{aligned}
& \text { Holding cost }=h_{s i} \int_{0}^{v_{i}} I_{s i}(t) d t \\
& H . C_{s}=h_{s i} \frac{\left(\alpha_{i}-\beta_{i} s_{i 2}\right)}{\theta}\left(\frac{e^{\theta v_{i}}-1}{\theta}-v_{i}\right)
\end{aligned}
$$

Ordering cost $=O_{i}$

$$
\begin{aligned}
& \text { Lost sale cost }=c_{2 i} \int_{v_{i}}^{T_{i} / n}\left(\alpha_{i}-\beta_{i} s_{i 2}\right) d t \\
& \text { L.S.C. } C_{i}=c_{2 i}\left(\alpha_{i}-\beta_{i} s_{i 2}\right)\left(\frac{T_{i}}{n}-v_{i}\right)
\end{aligned}
$$

Hence the total cost per unit time of the given inventory model as a function of $t_{1 i}, t_{2 i}, t_{3 i}, v_{i}$ and $T_{i}$ say T.A.C. $\left(t_{1 i}\right.$, $\mathrm{t}_{2 \mathrm{i}}, \mathrm{t}_{3 \mathrm{i}}, \mathrm{v}_{\mathrm{i}}, \mathrm{T}_{\mathrm{i}}$ ) is given by

$$
\begin{gathered}
\text { T.A.C. }=\frac{1}{T}\left\{c_{m i} a\left(\alpha_{i}-\beta_{i} s_{i 1}\right)\left(t_{3 i}-t_{2 i}\right)+c_{R i} b T_{i}\left(\alpha_{i}-\beta_{i} s_{i 1}\right)+P_{m i} a\left(t_{3 i}-t_{2 i}\right)\left(\alpha_{i}-\beta_{i} s_{i 1}\right)+\right. \\
P_{r i} a t_{1 i}\left(\alpha_{i}-\beta_{i} s_{i 1}\right)+K_{1 i}+K_{2 i}+K_{3 i}+s_{v i}\left\{\left(1-\gamma_{i}\right) b\left(\alpha_{i}-\beta_{i} s_{i 1}\right)\right\} T_{i}+ \\
h_{r i}\left\{\frac{(a-1)}{\theta}\left(\alpha_{i}-\beta_{i} s_{i 1}\right)\left(t_{1 i}+\frac{e^{-\theta t_{1 i}}-1}{\theta}\right)+\frac{\left(\alpha_{i}-\beta_{i} s_{i 1}\right)}{\theta}\left(\frac{e^{\theta\left(t_{2 i}-t_{1 i}\right)}-1}{\theta}\right)+\left(t_{1 i}-t_{2 i}\right)\right\}+h_{m i}\left\{\frac{(a-1)}{\theta}\left(\alpha_{i}-\beta_{i} s_{i 1}\right)\left\{\left(t_{3 i}-t_{2 i}\right)+\frac{e^{\theta\left(t_{2 i}-t_{3 i}\right)}-1}{\theta}\right\}+\right. \\
\left.\frac{\left(\alpha_{i}-\beta_{i} s_{i 1}\right)}{\theta}\left\{\left(\frac{e^{\theta\left(T_{i}-t_{3 i}\right)}-1}{\theta}\right)-\left(T_{i}-t_{3 i}\right)\right\}\right\}+h_{R i}\left\{\frac{(b-a)}{\theta}\left(\alpha_{i}-\beta_{i} s_{i 1}\right)\left(t_{1 i}-\frac{1-e^{\theta t_{1 i}}}{\theta}\right)+\right. \\
\left.b \frac{\left(\alpha_{i}-\beta_{i} s_{i 1}\right)}{\theta}\left\{\left(\frac{e^{\theta\left(t_{1 i}-T_{i}\right)}-1}{\theta}\right)+\left(T_{i}-t_{1 i}\right)\right\}\right\}+\frac{T_{i}}{n}\left[\left\{\frac{\left(\alpha_{i}-\beta_{i} s_{i 2}\right)}{\theta}\left(e^{\theta v_{i}}-1\right)+\left(\alpha_{i}-\beta_{i} s_{i 2}\right) \eta\left(\frac{T_{i}}{n}-v_{i}\right)\right\} c_{1 i}\right. \\
\left.h_{s i} \frac{\left(\alpha_{i}-\beta_{i} s_{i 2}\right)}{\theta}\left(\frac{e^{\theta v_{i}}-1}{\theta}-v_{i}\right)+O_{i}+c_{2 i}\left(\alpha_{i}-\beta_{i} s_{i 2}\right)\left(\frac{T_{i}}{n}-v_{i}\right)\right]
\end{gathered}
$$

Equation (32) denotes the cost function of the system in terms of $t_{1 i}, t_{2 i}, t_{3 i}, v_{i}$ and $T_{i}$. To find out the optimal solution of this system we have to find out the optimal values of $t_{1 i}, t_{2 i}, t_{3 i}, v_{i}$ and $T_{i}$. We have some relations 
between these variables.

$$
\begin{gathered}
0 \leq t_{1 i} \leq t_{2 i} \leq t_{3 i} \leq T_{i} \\
0 \leq v_{i} \leq T_{i} \\
(a-1)\left(1-e^{-\theta t_{1 i}}\right)=\left(e^{\theta\left(t_{2 i}-t_{1 i}\right)}-1\right) \\
(a-1)\left(1-e^{\theta\left(t_{2 i}-t_{3 i}\right)}\right)=\left(e^{\theta\left(T_{i}-t_{3 i}\right)}-1\right) \\
(b-a)\left(1-e^{\theta t_{1 i}}\right)=b\left(1-e^{\theta\left(t_{1 i}-T_{i}\right)}\right)
\end{gathered}
$$

Equations (33) and (34) are the essential conditions for the existence of this model. Equations (35) and (36) show the inventory level $I_{r}(t)$ and $I_{m}(t)$ at $t=t_{1}$ and $t=t_{3}$. Equation (37) demonstrates that the inventory level of collected and returned items will be the same at $\mathrm{t}=0$ and $\mathrm{t}=\mathrm{T}$.

Using the equations (35) - (37) the values of $t_{1 i}, t_{2 i}$ and $t_{3 i}$ can be find in the form of $T_{i}$, It can be said that

$$
\mathrm{t}_{1 \mathrm{i}}=\mathrm{f}_{1}\left(\mathrm{~T}_{\mathrm{i}}\right), \quad \mathrm{t}_{2 \mathrm{i}}=\mathrm{f}_{2}\left(\mathrm{~T}_{\mathrm{i}}\right), \quad \mathrm{t}_{3 \mathrm{i}}=\mathrm{f}_{3}\left(\mathrm{~T}_{\mathrm{i}}\right)
$$

Therefore the total average cost will be the function of variables $T_{i}$ and $v_{i}$.

\section{Numerical Example}

A numerical example is carried out to illustrate the model. Corresponding to the below mentioned parametric values the optimal value of time $\mathrm{v}_{\mathrm{i}}, \mathrm{T}_{\mathrm{i}}$ and T.A.C. $\mathrm{C}_{\mathrm{i}}$ are obtained for different three products.

Table 1.

\begin{tabular}{llll}
\hline Parameters & Product 1 & Product 2 & Product 3 \\
\hline $\boldsymbol{\alpha}_{\mathbf{i}}$ & 50 & 55 & 45 \\
$\gamma_{\mathbf{i}}$ & 0.4 & 0.45 & 0.35 \\
$\mathbf{s}_{\mathbf{i 1}}$ & 30 & 32 & 25 \\
$\mathbf{s}_{\mathbf{i} 2}$ & 40 & 41 & 43 \\
$\mathbf{a}$ & 1.5 & 1.6 & 1.4 \\
$\mathbf{b}$ & 0.4 & 0.45 & 0.35 \\
$\boldsymbol{\beta}_{\mathbf{i}}$ & 0.05 & 0.06 & 0.04 \\
$\mathbf{h}_{\mathbf{r i}}$ & 0.5 & 0.55 & 0.4 \\
$\mathbf{h}_{\mathbf{m i}}$ & 0.5 & 0.55 & 0.45 \\
$\mathbf{h}_{\mathbf{R i}}$ & 0.5 & 0.4 & 0.4 \\
$\mathbf{\theta}$ & 0.01 & 0.01 & 0.01 \\
$\mathbf{c}_{\mathbf{m i}}$ & 10 & 11 & 9 \\
$\mathbf{c}_{\mathbf{R i}}$ & 12 & 12.5 & 11.5 \\
$\mathbf{P}_{\mathbf{m i}}$ & 14 & 14.5 & 13.5 \\
$\mathbf{P}_{\mathbf{r i}}$ & 16 & 16.5 & 15.5 \\
$\mathbf{S}_{\mathbf{v i}}$ & 4 & 4.5 & 3.5 \\
$\mathbf{K}_{\mathbf{1 i}}$ & 500 & 500 & 500 \\
$\mathbf{K}_{\mathbf{2 i}}$ & 600 & 600 & 600 \\
$\mathbf{K}_{\mathbf{3 i}}$ & 700 & 700 & 700 \\
$\mathbf{c}_{\mathbf{1 i}}$ & 20 & 20 & 20 \\
$\mathbf{h}_{\mathbf{i s}}$ & 0.4 & 0.45 & 0.35 \\
$\mathbf{O}_{\mathbf{i}}$ & 250 & 300 & 200 \\
$\mathbf{n}$ & 5 & 5 & 5 \\
$\mathbf{v}_{\mathbf{i}}$ & $\mathbf{2 0 . 1 5 6 7}$ & $\mathbf{1 9 . 8 9 5 6}$ & $\mathbf{2 0 . 2 8 4 4}$ \\
$\mathbf{T}_{\mathbf{i}}$ & $\mathbf{2 3 . 7 4 3 9}$ & $\mathbf{2 3 . 4 2 0 3}$ & $\mathbf{2 3 . 9 0 2 1}$ \\
$\mathbf{T} . \mathbf{A .} \mathbf{C}_{\mathbf{i}}$ & $\mathbf{1 4 8 7 . 6 1}$ & $\mathbf{1 8 9 8 . 6 6}$ & $\mathbf{1 6 4 2 . 7}$ \\
\hline & & & \\
\hline & & &
\end{tabular}




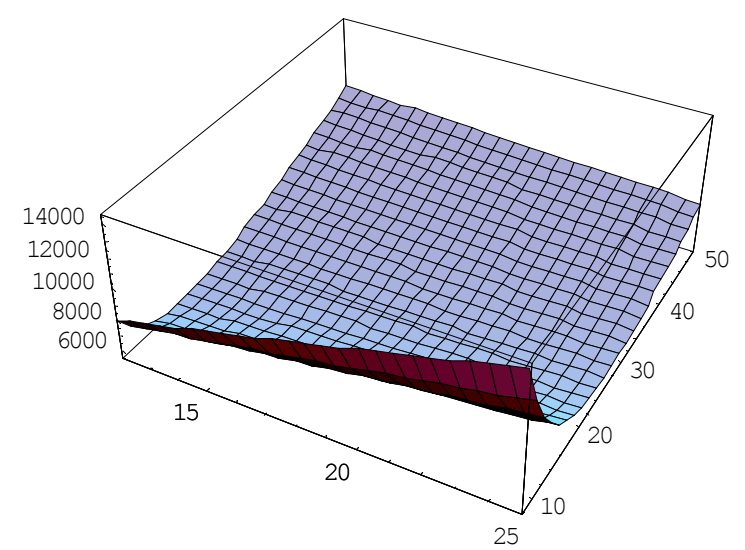

Figure 3. Convexivity of the T.A.C $_{1}$ function

\section{Sensitivity Analysis}

Corresponding to different associated parameters, a sensitivity analysis is carried out to check the stability of the model. The analysis has been done with the parameters $\theta, b, \beta_{1}, \gamma_{1}, \alpha_{1}, s_{1}$ and $a_{1}$ taking one parameter at a time and other variables unchanged and is shown in below mentioned tables.

Table 2. Sensitivity analysis with respect to deterioration parameter $(\theta)$ :

\begin{tabular}{ccccc}
\hline \% variation in $\theta$ & $\theta$ & $\mathrm{v}_{1}$ & $\mathrm{~T}_{1}$ & T.A.C $_{1}$ \\
\hline$-20 \%$ & 0.008 & 20.0962 & 23.6689 & 1128.13 \\
$-15 \%$ & 0.0085 & 20.1113 & 23.6876 & 1233.69 \\
$-10 \%$ & 0.009 & 20.1264 & 23.7063 & 1327.62 \\
$-5 \%$ & 0.0095 & 20.1416 & 23.7251 & 1411.78 \\
$0 \%$ & 0.01 & 20.1567 & 23.7439 & 1487.61 \\
$5 \%$ & 0.0105 & 20.1719 & 23.7627 & 1556.31 \\
$10 \%$ & 0.011 & 20.1872 & 23.7816 & 1618.86 \\
$15 \%$ & 0.0115 & 20.2024 & 23.8005 & 1676.05 \\
$20 \%$ & 0.012 & 20.2177 & 23.8194 & 1728.56 \\
\hline
\end{tabular}

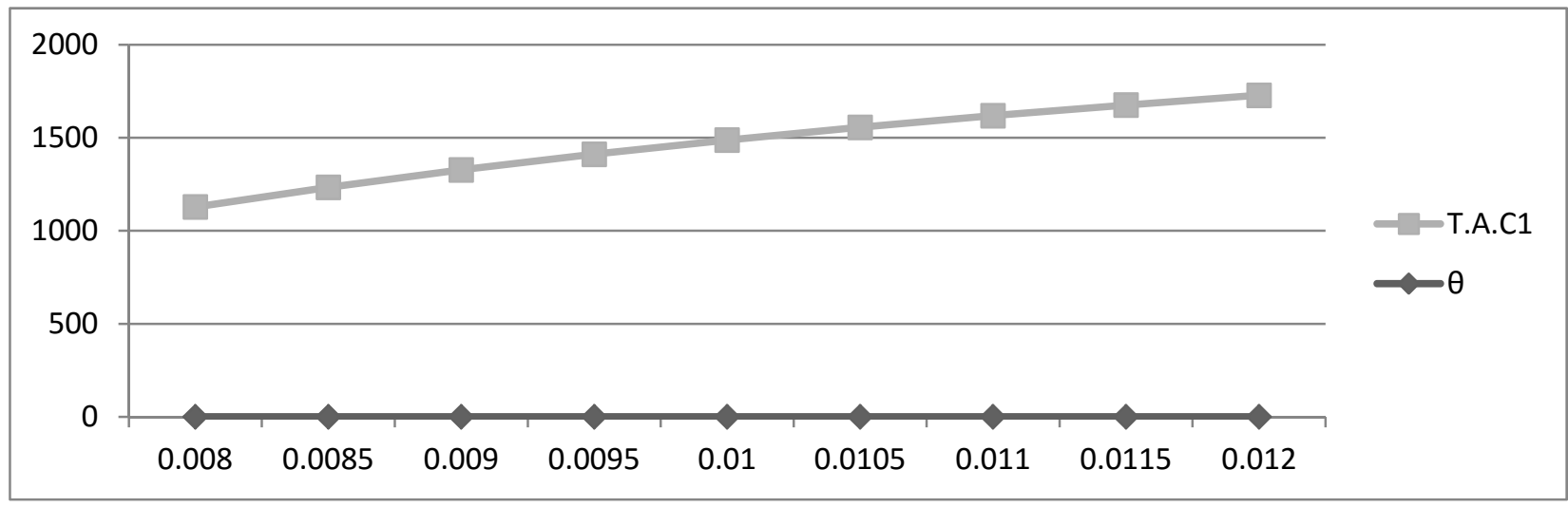

Figure 4. Variation in T.A.C $C_{1}$ with the variation in $\theta$ 
Table 3. Sensitivity analysis with respect to parameter b:

\begin{tabular}{ccccc}
\hline \% variation in b & $\mathbf{b}$ & $\mathbf{v}_{\mathbf{1}}$ & $\mathbf{T}_{\mathbf{1}}$ & $\mathbf{T}_{\mathbf{A}} \mathbf{A . \mathbf { C } _ { \mathbf { 1 } }}$ \\
\hline$-20 \%$ & 0.32 & 19.9468 & 23.4837 & 1739.47 \\
$-15 \%$ & 0.34 & 20.0018 & 23.5518 & 1672.5 \\
$-10 \%$ & 0.36 & 20.0552 & 23.618 & 1608.2 \\
$-5 \%$ & 0.38 & 20.1069 & 23.6821 & 1546.57 \\
$0 \%$ & 0.4 & 20.1567 & 23.7439 & 1487.61 \\
$5 \%$ & 0.42 & 20.2046 & 23.8032 & 1431.33 \\
$10 \%$ & 0.44 & 20.2503 & 23.8598 & 1377.75 \\
$15 \%$ & 0.46 & 20.2937 & 23.9136 & 1326.86 \\
$20 \%$ & 0.48 & 20.3346 & 23.9643 & 1278.67 \\
\hline
\end{tabular}

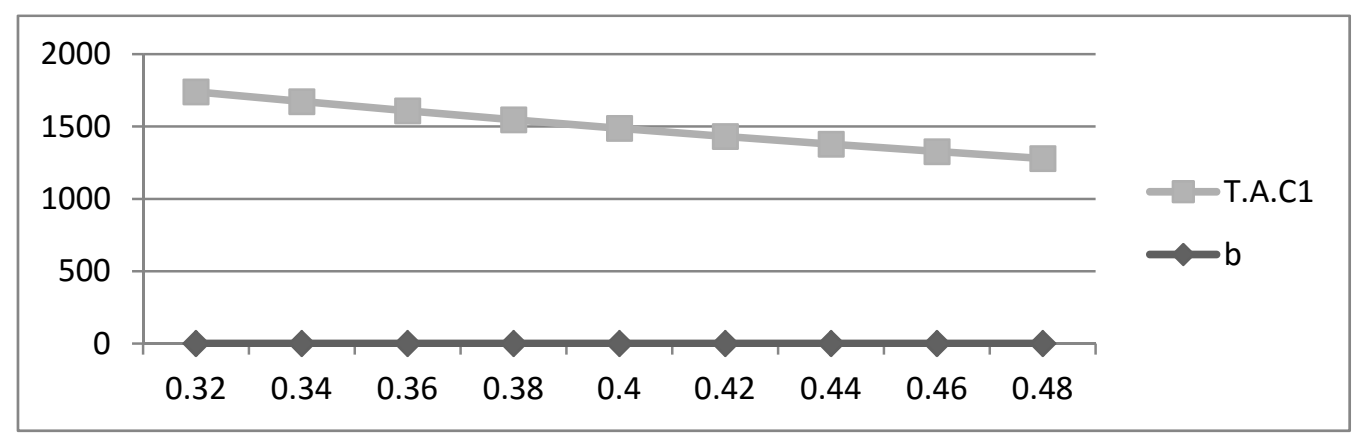

Figure 5. Variation in T.A. $C_{1}$ with the variation in $b$

Table 4. Sensitivity analysis with respect to demand parameter $\left(\beta_{1}\right)$ :

\begin{tabular}{clccc}
\hline \% variation in $\beta_{1}$ & \multicolumn{1}{c}{$\beta_{1}$} & \multicolumn{1}{c}{$\mathrm{v}_{1}$} & $\mathrm{~T}_{1}$ & T.A.C $\mathrm{C}_{1}$ \\
\hline$-20 \%$ & 0.04 & 20.148 & 23.7331 & 1494.73 \\
$-15 \%$ & 0.0425 & 20.1502 & 23.7358 & 1492.95 \\
$-10 \%$ & 0.045 & 20.1523 & 23.7385 & 1491.17 \\
$-5 \%$ & 0.0475 & 20.1545 & 23.7412 & 1489.39 \\
$0 \%$ & 0.05 & 20.1567 & 23.7439 & 1487.61 \\
$5 \%$ & 0.0525 & 20.1589 & 23.7466 & 1485.83 \\
$10 \%$ & 0.055 & 20.1611 & 23.7494 & 1484.05 \\
$15 \%$ & 0.0575 & 20.1634 & 23.7521 & 1482.27 \\
$20 \%$ & 0.06 & 20.1656 & 23.7549 & 1480.48 \\
\hline
\end{tabular}

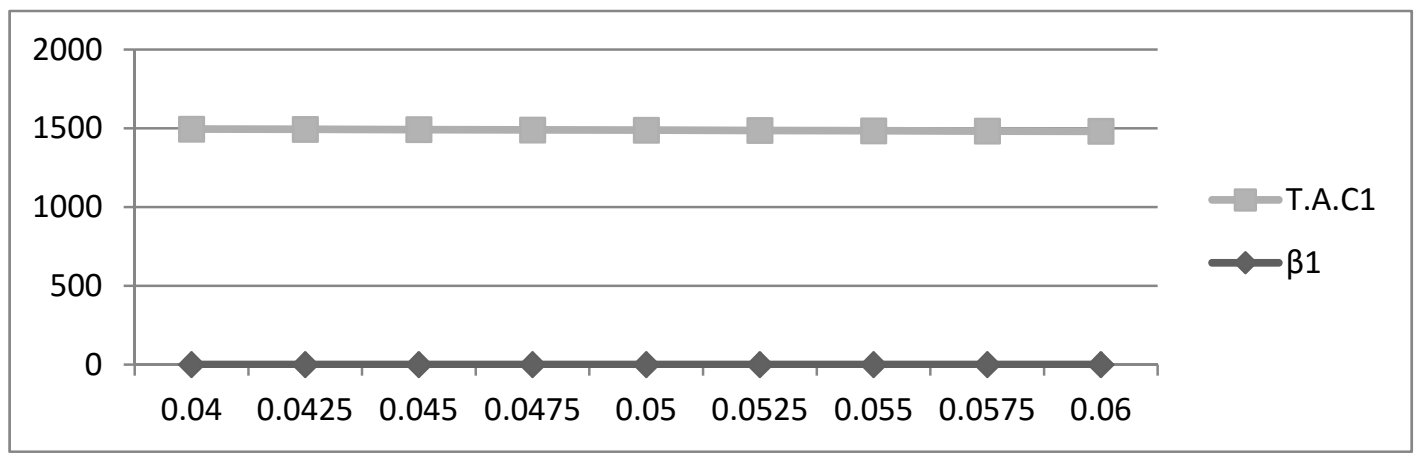

Figure 6. Variation in T.A.C $C_{1}$ with the variation in $\beta_{1}$ 
Table 5. Sensitivity analysis with respect to parameter $\gamma_{1}$ :

\begin{tabular}{ccccc}
\hline \% variation in $\gamma_{\mathbf{1}}$ & $\boldsymbol{\gamma}_{\mathbf{1}}$ & $\mathbf{v}_{\mathbf{1}}$ & $\mathbf{T}_{\mathbf{1}}$ & $\mathbf{T . A . C}_{\mathbf{1}}$ \\
\hline$-20 \%$ & 0.32 & 20.1567 & 23.7439 & 1481.4 \\
$-15 \%$ & 0.34 & 20.1567 & 23.7439 & 1482.95 \\
$-10 \%$ & 0.36 & 20.1567 & 23.7439 & 1484.5 \\
$-5 \%$ & 0.38 & 20.1567 & 23.7439 & 1486.06 \\
$0 \%$ & 0.4 & 20.1567 & 23.7439 & 1487.61 \\
$5 \%$ & 0.42 & 20.1567 & 23.7439 & 1489.16 \\
$10 \%$ & 0.44 & 20.1567 & 23.7439 & 1490.71 \\
$15 \%$ & 0.46 & 20.1567 & 23.7439 & 1492.26 \\
$20 \%$ & 0.48 & 20.1567 & 23.7439 & 1493.82 \\
\hline
\end{tabular}

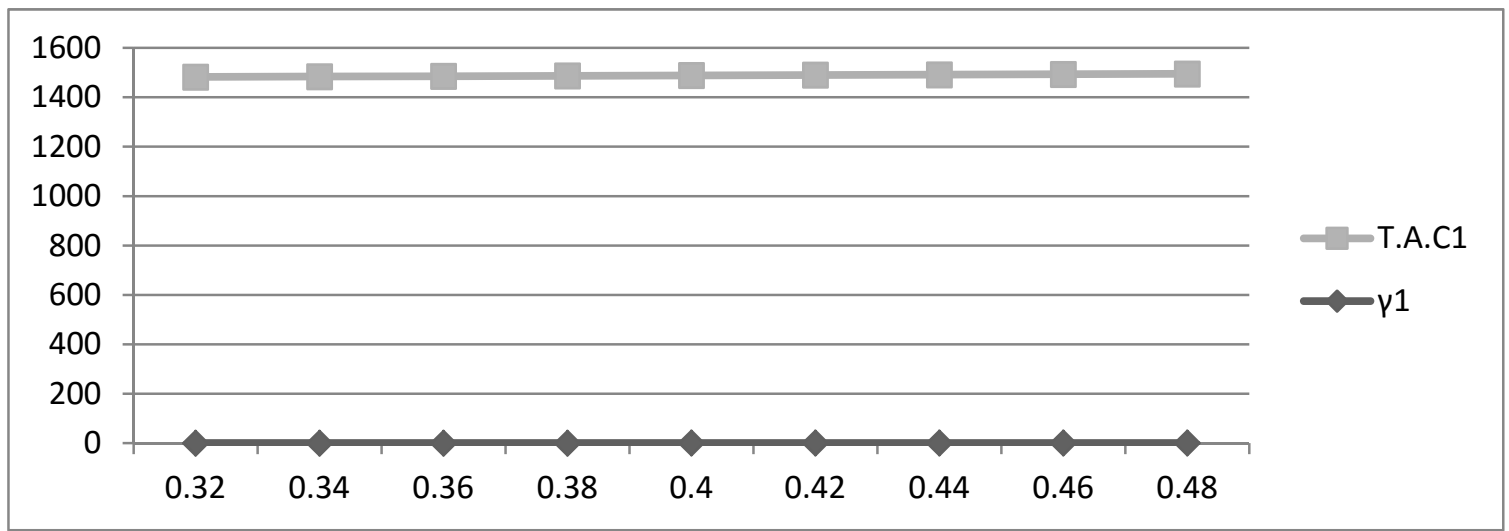

Figure 7. Variation in T.A.C1 with the variation in $\gamma 1$

Table 6 . Sensitivity analysis with respect to demand parameter $(\alpha 1)$ :

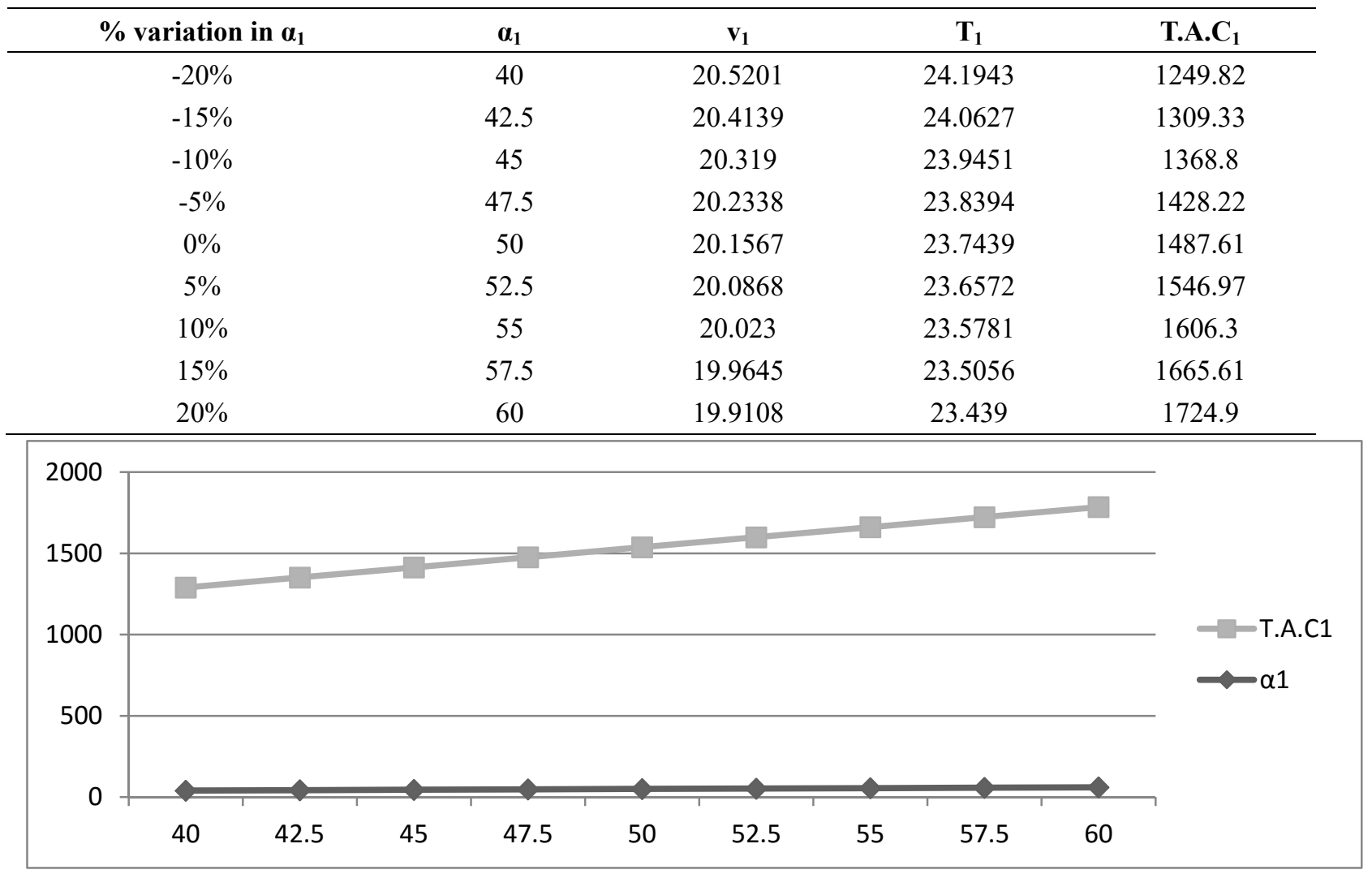

Figure 8. Variation in T.A.C1 with the variation in $\alpha 1$ 
Table 7. Sensitivity analysis with respect to selling price (s11)

\begin{tabular}{ccccc}
\hline \% variation in $\mathrm{s}_{11}$ & $\mathrm{~s}_{11}$ & $\mathrm{v}_{1}$ & $\mathrm{~T}_{1}$ & T.A.C $\mathrm{C}_{1}$ \\
\hline$-20 \%$ & 24 & 20.148 & 23.7331 & 1494.73 \\
$-15 \%$ & 25.5 & 20.1502 & 23.7358 & 1492.95 \\
$-10 \%$ & 27 & 20.1523 & 23.7385 & 1491.17 \\
$-5 \%$ & 28.5 & 20.1545 & 23.7412 & 1489.39 \\
$0 \%$ & 30 & 20.1567 & 23.7439 & 1487.61 \\
$5 \%$ & 31.5 & 20.1589 & 23.7466 & 1485.83 \\
$10 \%$ & 33 & 20.1611 & 23.7494 & 1484.05 \\
$15 \%$ & 34.5 & 20.1634 & 23.7521 & 1482.27 \\
$20 \%$ & 36 & 20.1656 & 23.7549 & 1480.48 \\
\hline
\end{tabular}

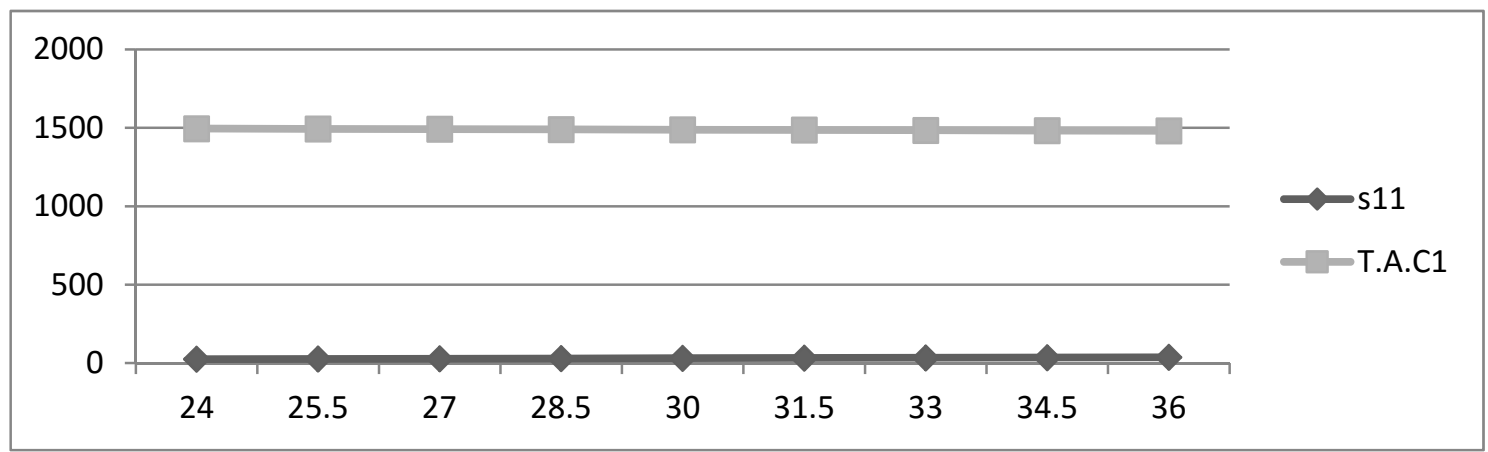

Figure 9. Variation in T.A. $C_{1}$ with the variation in $s_{1}$

Table 8 . Sensitivity analysis with respect to production parameter (a1):

\begin{tabular}{ccccc}
\hline \% variation in $\mathrm{a}_{1}$ & $\mathrm{a}_{1}$ & $\mathrm{v}_{1}$ & $\mathrm{~T}_{1}$ & $\mathrm{~T}$ A.C \\
\hline$-20 \%$ & 1.2 & 20.6989 & 24.4159 & 1592.02 \\
$-15 \%$ & 1.275 & 20.5379 & 24.2164 & 1561.34 \\
$-10 \%$ & 1.35 & 20.3959 & 24.0404 & 1534.98 \\
$-5 \%$ & 1.425 & 20.2697 & 23.8839 & 1509.62 \\
$0 \%$ & 1.5 & 20.1567 & 23.7439 & 1487.61 \\
$5 \%$ & 1.575 & 20.0551 & 23.6179 & 1467.68 \\
$10 \%$ & 1.65 & 19.9632 & 23.504 & 1449.56 \\
$15 \%$ & 1.725 & 19.8797 & 23.4005 & 1433.01 \\
$20 \%$ & 1.8 & 19.8034 & 23.306 & 1417.83 \\
\hline
\end{tabular}

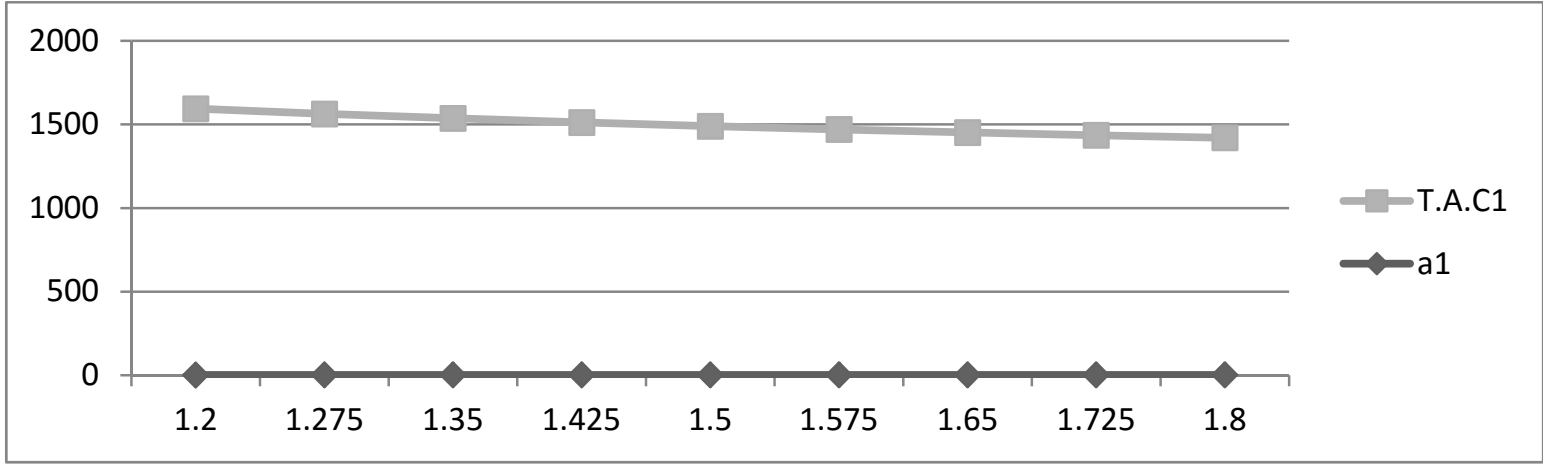

Figure 10. Variation in T.A.C1 with the variation in a1

\subsection{Observations}

Table 2 shows the variation in T.A.C. with the variation in deterioration parameter ' $\theta$ '. From this table it is 
observed that with the increment in deterioration rate the T.A.C. of the system increases.

1. From table 3 it is observed that an increment in parameter ' $b$ ' results a decrease in T.A.C. of the system.

2. Table 4 lists the variation in demand parameter $\left(\beta_{1}\right)$. It is observed from this table that as the value of ' $\beta_{1}$ ' increases, the T.A.C. of the system shows the reverse effect.

3. Table 5 and table 6 show the variation in parameter ' $\gamma_{i}$ ' and in demand parameter ' $\alpha_{i}$ ' and from these it is observed that an increment in both the parameters result an increment in T.A.C.

4. Table 7 and table 8 show the variation in T.A.C. with the changes in selling price ' $\mathrm{s}_{1}$ ' and production parameter 'a' respectively. It is observed that an increment in selling price and production parameter result a decline in T.A.C.

\section{Conclusion}

In this paper we have presented an integrated production inventory model for multi items. This is a closed loop supply chain, introduced with the production of new items and remanufacturing of collected and returned items with deterioration. The demand rate is taken as a function of selling price which shows a very realistic phenomenon. A numerical example is shown to illustrate the model. The model is optimized and the convexity of the model is shown. A sensitivity analysis is also performed to check the stability of the model. For future scope the model can be extended for stochastic demand rate and with learning and forgetting effects for production and manufacturing.

\section{References}

Balkhi, Z. T. (2009). Multi- item production inventory systems with budget constraints. Proceedings of the1stInternational Conference on manufacturing Engineering, Quality and Production Systems (MEQAPS'09), 232-241.

Ben-Daya, M., \& Raouf, A. (1993). On the constrained multi-item single-period inventory Problem. International journal of Production Management, 13, 104-112.

Bhattacharya, D. K. (2005). Production, manufacturing and logistics on multi-item inventory. European Journal of Operational Researc, 162, 786-791.

Hadidi, L. A., Turki, U. M. A., \& Rahim, M. A. (2011). An integrated cost model for production scheduling and perfect maintenance. International Journal of Mathematics in Operational Research, 3(4), 395-413.

Lenard, J. D., \& Roy, B. (1995). Multi-item inventory control: A multi-criteria view. European Journal of Operational Research, 87, 685-692.

Maity, K., \& Maiti, M. (2009). Optimal inventory policies for deteriorating complementary and substitute items, International Journal of Systems Science, 40, 267-276.

Park, K. S. (1983). An integration production inventory model for decaying raw materials. International Journal of Systems Science, 14, 801-806.

Saxena, P., \& Singh, S. R. (2013). A two -warehouse production inventory model with variable demand and permissible delay in payment under inflation. International Journal of soft computing and Engineering, 3(5), 145-161.

Saxena, P., \& Singh, S. R. (2014). Fuzzy warehouse inventory model for items with imperfect quality under trade credit policy and inflationary conditions. Innovative Applications of Computational Intelligence on Power, Energy and Controls with their impact on Humanity (CIPECH), Ghaziabad, 69-74.

Schrady, D. A. (1967). A deterministic inventory model for repairable items Naval Research Logistics Quarterly, 14, 391-398.

Singh, N., Vaish, B., \& Singh, S. R. (2010). An EOQ model with Pareto distribution for deterioration, Trapezoidal type demand and backlogging under trade credit policy. The IUP Journal of Computational Mathematics, 3(4), 30-53.

Singh, S. R., \& Prasher, L. (2014). A production inventory model with flexible manufacturing, random machine breakdown and stochastic repair time. International Journal of Industrial Engineering Computations, 5 (4), 575-588.

Singh, S. R, Sharma, R., \& Chauhan, A. (2014). Two echelon supply chain model for deteriorating items with effective investment in preservation technology. International Journal Mathematics in Operational Research, 6(1), 78-99. 
Singh, S. R., Sharma, R., \& Singh, A. P. (2015). An EPQ model for non-instantaneous deteriorating item with time dependent holding cost and exponential demand rate. International Journal of Operational Research, 23(2), 145-161.

Singh, S. R., Gupta, V., \& Bansal, P. (2013). EOQ model with volume agility, variable demand rate, Weibull deterioration rate and inflation.International Journal of Computer Applications, 72(23), 1-6.

Singh, S. R., Chauhan, A., \& Sharma, R. (2014). A deteriorating production inventory problem with space restriction. Journal of Information \& Optimization Sciences, 35(3), 203-229.

Singh, S. R., Khurana, D., \& Tayal, S. (2016). An economic order quantity model for deteriorating products having stock dependent demand with trade credit period and preservation technology. Uncertain Supply Chain Management, 4(1), 29-42.

Singh, S. R., \& Singh, N. (2013). Green supply chain model with product remanufacturing under volume flexible environment. Procedia Technology, 10, 216-226.

Singh, S. R., \& Diksha. (2009). Integrated vendor-buyer cooperative model with multivariate demand and progressive credit period. Journal of Operations Management, 8(2), 36-50.

Singhal, S., \& Singh, S. (2013). Volume flexible multi items inventory system with imprecise environment. International Journal of Industrial Engineering Computations, 4(4), 457-468.

Soni, H. N., \& Patel, K. A. (2014). Optimal policies for vendor-buyer inventory system involving defective items with variable lead time and service level constraint. International Journal of Mathematics in Operational Research, 6(3), 316-343.

Tayal, S, Singh S. R., \& Sharma, R. (2014). An inventory model for deteriorating items with seasonal products and an option of an alternative market. Uncertain Supply Chain Management, 3, 69-86.

Tayal, S., Singh S. R., \& Sharma, R. (2014). A multi item inventory model for deteriorating items with expiration date and allowable shortages. Indian Journal of Science and Technology, 7(4), 463-471.

Tayal, S., Singh, S. R., \& Sharma, R. (2016). An integrated production inventory model for perishable products with trade credit period and investment in preservation technology. International Journal of Mathematics in Operational Research, 8(2), 137-163.

Teunter, R. H. (2001). Economic ordering quantities for recoverable item inventory system. Naval Research Logistics, 48, 484-495.

Yadav, D., Pundir, S., \& Kumari, R. (2011). A fuzzy multi item production model with reliability and flexibility under limited storage capacity with deterioration via geometric programming. International Journal of Mathematics in Operational Research, 3(1), 78-98.

Yan, H., \& Cheng, T. C. E. (1998). Optimal production stopping and restarting times for an EOQ model with deteriorating items. Journal of the Operational Research Society, 49, 1288-1295.

\section{Copyrights}

Copyright for this article is retained by the author(s), with first publication rights granted to the journal.

This is an open-access article distributed under the terms and conditions of the Creative Commons Attribution license (http://creativecommons.org/licenses/by/3.0/). 